\title{
Orbitotomías óseas mediante abordaje coronal: estudio retrospectivo de 87 lesiones
} intraorbitarias

\author{
P. Infante-Cossío; M. Molina-Martínez; A. García-Perla; F. Espín-Gálvez*; J. Romero Arce** y J.L. Gutiérrez- \\ Pérez
}

Servicio de Cirugía Maxilofacial. Hospital Universitario Virgen del Rocío, Sevilla. *Servicio de Cirugía Maxilofacial. Complejo Hospitalario Torrecárdenas, Almería. **Servicio de Neurocirugía. Hospital Universitario Virgen del Rocío, Sevilla.

\section{Resumen}

Objetivo. Presentar nuestra experiencia en 80 pacientes con tumores y lesiones intraorbitarias en los que se realizaron 87 orbitotomías óseas mediante abordaje coronal, durante un período de 12 años.

Material, métodos y resultados. El diagnóstico histológico más frecuente fue el hemangioma, y la localización anatómica más frecuente la retrobulbar intracónica. La presentación bilateral de lesiones ocurrió en 7 pacientes. Las orbitotomías más utilizadas fueron la lateral y supero-lateral $(\mathbf{8 7 , 3 5 \%})$. En un $\mathbf{9 0 , 8 0 \%}$ de los casos se realizó algún tipo de osteosíntesis, un 51,75\% con placas bio-reabsorbibles. La exposición o rotura de la duramadre intraoperatoria ocurrió en 10 pacientes. Se recogieron 45 complicaciones postoperatorias transitorias y 21 permanentes, las más frecuentes fueron las nerviosas (diplopia y ptosis) que se resolvieron espontáneamente en su mayoría en un corto intervalo de tiempo. En 15 pacientes se requirió colaboración multidisciplinar con neurocirugía.

Conclusiones. La incisión coronal permite realizar cualquier orbitotomía ósea con seguridad, garantía y buen resultado estético y funcional. Las orbitotomías lateral y supero-lateral proporcionan un abordaje ideal extradural al espacio retrobulbar. El acceso al ápex, canal orbitario y zona medial nervio óptico requiere comúnmente un abordaje combinado neuroquirúrgico a través de fosa craneal anterior. La fijación de las orbitotomías mediante osteosíntesis biorreabsorbible constituye una alternativa a las placas de titanio, incluso puede ser de primera elección en la edad pediátrica. La morbilidad de esta cirugía es baja.

PALABRAS CLAVE: Órbita. Tumor. Cirugía de órbita. Incisión bicoronal. Orbitotomía. Placa reabsorbible.

Osseous orbitotomies using a coronal flap: A retrospective study of 87 intraorbital lesions

Recibido: 11-01-08. Aceptado: 13-03-08

\section{Summary}

Objective. To present our experience in 80 patients with intraorbital tumours and lesions who underwent 87 osseous orbitotomies with coronal incision during a 12 year period.

Material, methods and results. Hemangioma was the most frequent histologic diagnosis, being the intraconical retrobulbar compartment the most common location. Bilateral presentation of lesions occurred in 7 patients. Lateral and supero-lateral orbitotomies were preferably applied $(\mathbf{8 7 . 3 5 \% )}$ ). In $\mathbf{9 0 . 8 0 \%}$ of cases some kind of osteosynthesis was employed, $51.75 \%$ with bio-resorbable plates. Intraoperative exposure or dura mater breakage occurred in 10 patients. 45 transitory and 21 permanent postoperative complications were seen, being the nervous ones (diplopia and ptosis) the most frequent. Most of these were spontaneously solved in a short time period. 15 patients required multi-disciplinary collaboration with neurosurgery.

Conclusions. Coronal incision allows any bone orbitotomy, with security, guarantee and good aesthetic and functional results. Lateral and supero-lateral orbitotomies provide an ideal extradural approach to the retrobulbar compartment. An approach to the apex, orbital channel and medial compartment to the optic nerve, usually requires a combined neurosurgical approach through anterior cranial fossa. Orbitotomy fixation with bio-resorbable ostheosynthesis is an alternative to titanium plates. They can even be a first choice in paediatric age. The morbidity of this surgical technique is low.

KEY WORDS: Orbit. Tumour. Orbital surgery. Bicoronal incision. Orbitotomy. Resorbable plate.

Introducción

La cirugía de los tumores y lesiones de la órbita suele presentar un alto grado de complejidad. El abordaje quirúrgico y orbitotomía vendrá condicionado de un lado por la 
Tabla 1

Manifestaciones clínicas

\begin{tabular}{lcc}
\hline & $\mathrm{n}$ & $\%$ \\
\hline Proptosis unilateral & 84 & 93,10 \\
Dolor & 51 & 58,62 \\
Conjuntivitis / queratitis & 17 & 19,54 \\
Ptosis palpebral & 14 & 16,09 \\
Disminución agudeza visual & 14 & 16,09 \\
Aumento presión intraocular & 10 & 11,49 \\
Masa & 8 & 9,19 \\
Diplopia & 8 & 9,19 \\
Proptosis bilateral & 3 & 3,44 \\
Asintomáticos & 3 & 3,44 \\
\hline
\end{tabular}

Tabla 2

Localización anatómica y topográfica

\begin{tabular}{lcc}
\hline & $\mathrm{n}$ & $\%$ \\
\hline Órbita izquierda & 42 & 48,30 \\
Orbita derecha & 45 & 51,70 \\
$\quad$ Total & 87 & \\
\hline Retrobulbar & 53 & 60,90 \\
$\quad$ Extracónico & 8 & 9,20 \\
$\quad$ Intracónico & 43 & 49,40 \\
$\quad$ Extra / Intra & 2 & 2,30 \\
\hline Ante + retrobulbar & & \\
$\quad$ Extracónico & 13 & 14,95 \\
$\quad$ Intracónico & 9 & 9,20 \\
$\quad$ Extra / Intra & 0 & \\
& 4 & 4,30 \\
Con extensión paraorbitaria & & \\
$\quad$ S.N.C. & 21 & 24,15 \\
$\quad$ Seno /Hueso Frontal & 4 & 4,30 \\
$\quad$ Seno etmoidal & $1 / 5$ & 6,60 \\
$\quad$ Seno esfenoidal & 3 & 3,45 \\
$\quad$ Seno maxilar & 4 & 4,30 \\
\hline
\end{tabular}

clínica, histología, localización y extensión de cada tumor, y de otro lado por las posibilidades exeréticas y reconstructivas. Se han ideado y desarrollado numerosos tipos de abordajes, orbitotomías óseas y osteotomías orbitarias para acceder a las diferentes regiones orbitarias y paraorbita- rias ${ }^{11,12,14-16}$, cuya indicación dependerá fundamentalmente de la localización de la lesión tumoral y de los objetivos del tratamiento. En general se admite que los tumores localizados por delante del ecuador del globo ocular se pueden extirpar por vía anterior sin precisar de osteotomía, mientras que el abordaje de los tumores que se sitúan retroecuatoriales respecto al globo ocular es más controvertido. La mayor parte de los tumores y lesiones retroecuatoriales precisan de algún tipo de osteotomía y de abordajes suficientemente amplios para poder garantizar un acceso adecuado y seguro para realizar la remoción tumoral con la menor tasa de complicaciones. Entre las diferentes vías de abordaje descritas en cirugía cráneo-facial, la más importante es el abordaje coronal ${ }^{1,10,23}$. La orbitotomía lateral es la técnica estándar para la extirpación o la biopsia de lesiones intraorbitarias laterales al nervio óptico ${ }^{11,14}$. La reciente introducción de las osteosíntesis de los fragmentos óseos orbitarios con material bio-reabsorbible ha abierto nuevas perspectivas en la cirugía de la órbita ${ }^{5}$.

El objetivo de este trabajo ha sido presentar nuestra experiencia clínica y terapéutica en 80 pacientes con tumores y lesiones orbitarias en los que se realizaron 87 orbitotomías óseas mediante un abordaje coronal, durante un período de 12 años.

\section{Material y métodos}

Durante el período comprendido entre enero de 1991 y diciembre de 2002 se realizaron 87 orbitotomías óseas en 80 pacientes con tumores o lesiones orbitarias remitidos al Servicio de Cirugía Maxilofacial de los Hospitales Universitarios Virgen del Rocío de Sevilla. 30 pacientes (37,5\%) fueron hombres y $50(62,5 \%)$ mujeres (proporción mujeres/hombres de 1,7/1). La media edad fue de 34,05 años, con un rango de edad que comprendió desde los 3 a los 84 años. El motivo de consulta más frecuente fue la proptosis unilateral, presente en el $93,10 \%$ de los pacientes (Tabla 1). En todos los pacientes se realizó un estudio con TC de ambas órbitas. La RNM se solicitó en un segundo tiempo en 40 pacientes $(45,95 \%)$ cuando se necesitaron más datos diagnósticos de localización y/o extensión, para estudio del nervio óptico, afectación intracraneal o sospecha de meningioma. La arteriografía se llevó a cabo en 6 pacientes $(6,60 \%)$ ante la sospecha de lesiones de origen vascular. La órbita derecha se afectó en el $51,70 \%$ de los pacientes y la izquierda en el 48,30\% (Tabla 2). La localización anatómica intraorbitaria fue: retrobulbar en el $60,90 \%$, ante-retrobulbar en el $14,95 \%$ y con extensión paraorbitaria en el $24,15 \%$. La localización anatómica más frecuente fue la retrobulbar intracónica en el 49,40\%.

Se registraron 87 tumoraciones y lesiones orbitarias en 80 pacientes, de las que 77 fueron tumores de órbita (15 se consideraron formas histológicas malignas, 14 benig- 


\begin{tabular}{|c|c|c|}
\hline \multicolumn{3}{|c|}{$\begin{array}{c}\text { Tabla } 3 \\
\text { Tipos histopatológicos }\end{array}$} \\
\hline & $\mathrm{n}$ & $\%$ \\
\hline Tumores y lesiones orbitarias & 77 & 88,50 \\
\hline Hemangioma & 22 & 25,30 \\
\hline Meningioma meningotelial & 14 & 16,10 \\
\hline Rabdomiosarcoma & 12 & 13,80 \\
\hline Mucocele & 4 & 4,60 \\
\hline Glioma óptico & 4 & 4,60 \\
\hline Fibrolipoma & 3 & 3,45 \\
\hline Neurobibroma & 3 & 3,45 \\
\hline Carcinoma adenoide-quístico & 2 & 2,30 \\
\hline Malformación vascular & 2 & 2,30 \\
\hline Ganglioneuroblastoma & 1 & 1.15 \\
\hline Schwanoma & 1 & 1.15 \\
\hline Quiste dermoide & 1 & 1.15 \\
\hline Granuloma eosinófilo & 1 & 1.15 \\
\hline Tumor seno endodérmico & 1 & 1.15 \\
\hline Osteoma & 1 & 1.15 \\
\hline Osteocondroma & 1 & 1.15 \\
\hline Adenoma pleomorfo & 1 & 1.15 \\
\hline Linfoma no Hodgkin & 1 & 1.15 \\
\hline Carcinoma epidermoide & 1 & 1.15 \\
\hline Hematoma & 1 & 1.15 \\
\hline Procesos expansivos & 10 & 11,50 \\
\hline Enfermedad de Graves-Basedow & 6 & 6,90 \\
\hline Pseudotumor inflamatorio & 4 & 6,90 \\
\hline
\end{tabular}

nas con afectación o destrucción de estructuras nobles oculares y 48 benignas), y 10 procesos expansivos propiamente no tumorales, pero de comportamiento clínico agresivo (Tabla 3). La presentación bilateral de lesiones ocurrió en 7 pacientes ( 2 hemangiomas, 2 neurofibromas, 2 pseudotumores inflamatorios y 1 linfoma no Hodgkin). El hemangioma fue el diagnostico histológico más frecuente en el $25,30 \%$.

Se consideraron tres tipos de indicación quirúrgica para las orbitotomías:

1. Exéresis tumoral, en lesiones intraorbitarias de localización retrobulbar, ante-retrobulbar o con extensión paraorbitaria, y lesiones intraóseas con afectación de la pared orbitaria. En las lesiones benignas se realizó una extirpación conservadora respetando estructuras nobles intraorbitarias. En las lesiones malignas se planteó una

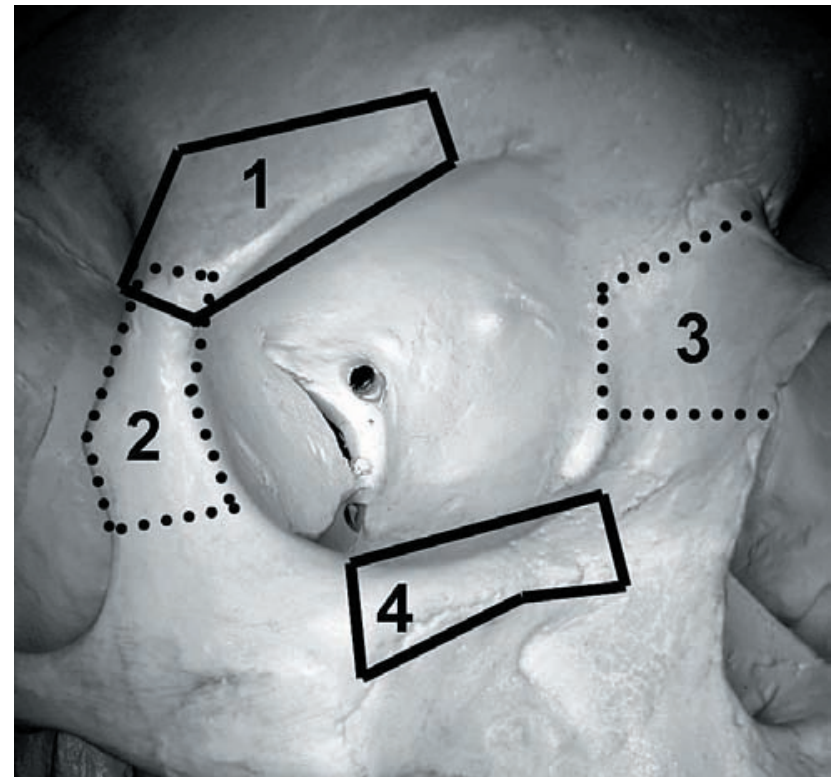

Figura 1. Esquema de diferentes orbitotomías según localización: (1) orbitotomía superior, (2) orbitotomía lateral, (3) orbitotomía medial, y (4) orbitotomía del suelo de órbita.

exéresis tumoral radical amplia según los requerimientos de histología, localización y extensión.

2. Biopsia diagnóstica, en lesiones cuya clínica y pruebas de imagen no permitían un diagnóstico de presunción. Se realizó biopsia del material tumoral y valoración de extensión tumoral.

3. Descompresión orbitaria, para disminuir con carácter urgente o profiláctico la presión intraorbitaria.

La planificación del tipo de orbitotomía varió según la localización topográfica de la lesión:

1. Orbitotomía lateral/superolateral, para tumores retrobulbares laterales, intra e extracónicos.

2. Orbitotomía lateral/superolateral combinadas $o$ no con vía neuroquirúrgica intracraneal de fosa craneal anterior, para tumores cercanos al ápex, mediales al nervio óptico o del canal óptico.

3. Orbitotomía medial, para tumores retrobulbares mediales.

4. Orbitotomía del suelo de órbita, para tumores del suelo orbitario o con afectación del seno maxilar.

\section{Técnica quirúrgica}

Mediante una incisión bi-coronal se realiza el acceso a la región periorbitaria, con despegamiento subperióstico y liberación del reborde supraorbitario, raíz del arco cigomático y pared lateral de la órbita. En caso de acceso a la pared medial, se realiza además un despegamiento subperióstico cuidadoso de la raíz de la pirámide nasal, para facilitar el despegamiento de los rebordes orbitarios inferiores. Se 


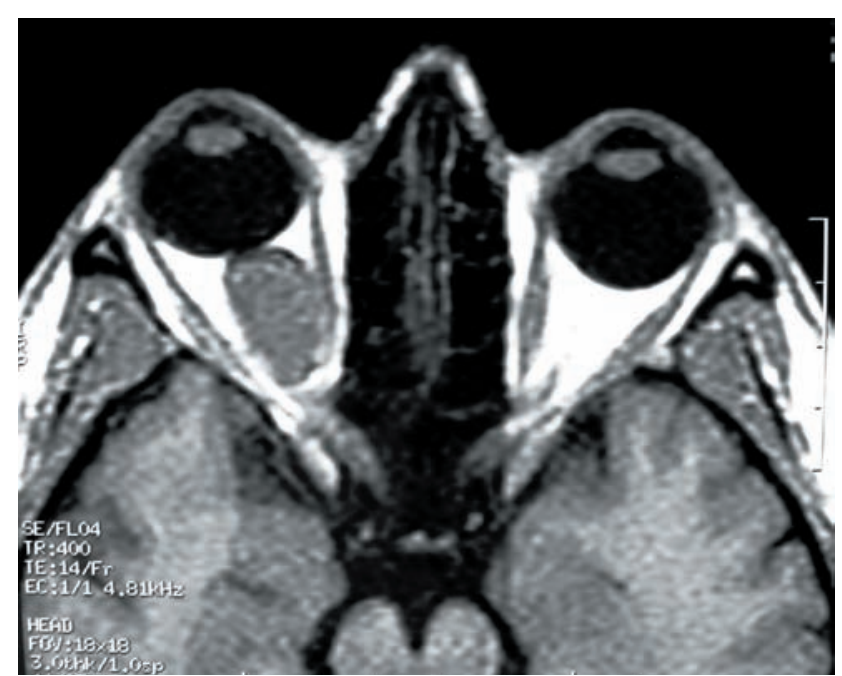

Figura 2 A. Caso 1. RNM que muestra una lesión redondeada y bien definida en el espacio retrobulbar.

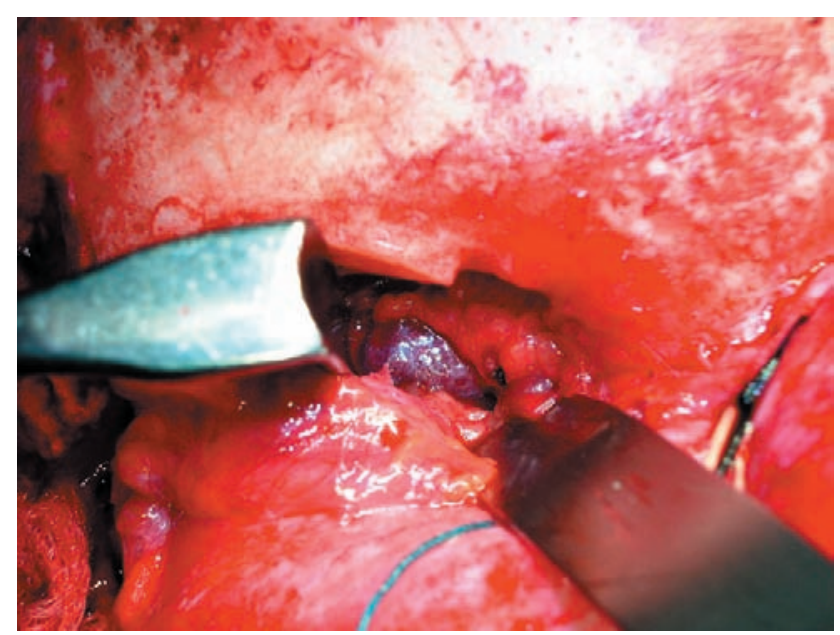

Figura 2 C. Caso 1. Tras realizar la orbitotomía superolateral y entrar en la periórbita, aparece un hemangioma cavernoso que se extirpa en bloque.

libera el paquete supraorbitario y el paquete supratroclear.

El diseño de la osteotomía depende de la localización de la lesión (Fig. 1). En la orbitotomía superior, la osteotomía comienza con un trazo horizontal que se inicia lateral a la escotadura supraorbitaria, quedando confinada al espesor del reborde orbitario del hueso frontal. En la mayoría de las ocasiones se amplía hasta el reborde óseo superolateral. En la osteotomía superolateral, el trazo horizontal se continúa con un trazo vertical realizado en la pared lateral de la órbita. El límite inferior de esta osteotomía suele situarse algo por debajo de la sutura frontomalar. En la orbitotomía lateral, la osteotomía se hace por encima de la unión frontocigomática. El corte inferior puede prolongarse hasta la fisura orbitaria inferior y hacia atrás puede extenderse tanto como se considere necesario. Si es preciso eliminar

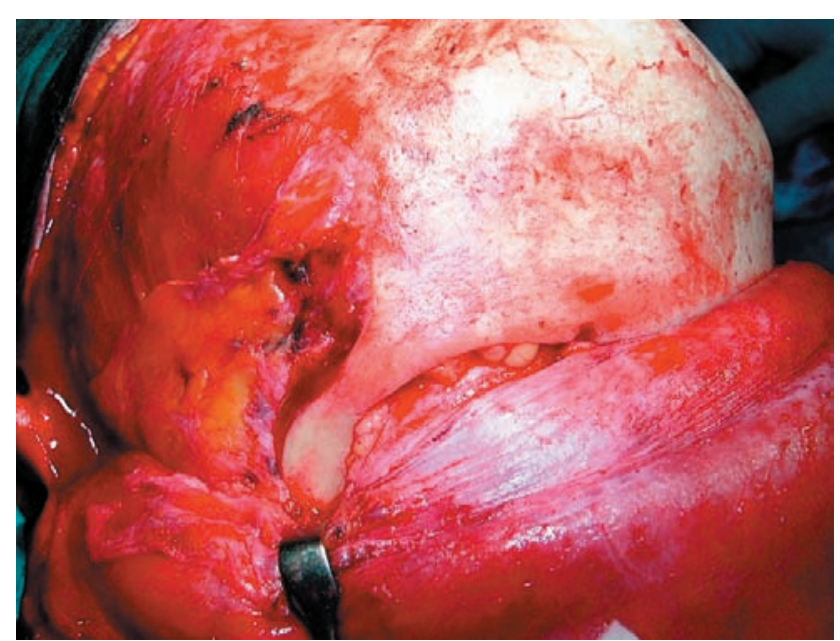

Figura 2 B. Caso 1. Diseño del colgajo coronal, disección de los dos planos y despegamiento parcial del músculo temporal, exponiendo el contorno de la órbita.

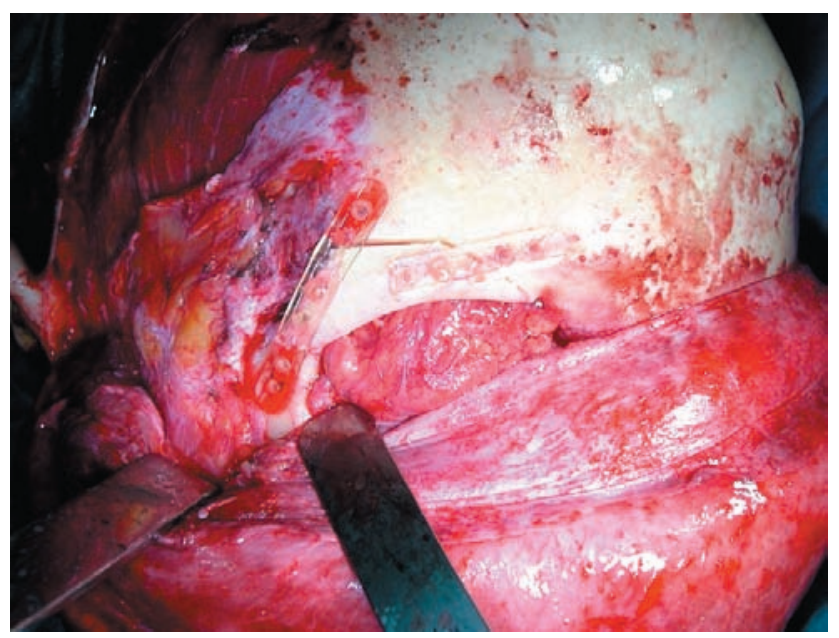

Figura 2 D. Caso 1. Reposición del fragmento osteomizado y fijación con dos placas reabsorbibles.

el hueso de la pared lateral de la órbita (ala mayor del esfenoides) se realiza ostectomía pero sin penetrar endocranealmente. En la orbitotomía lateral y superolateral es útil despegar parcialmente el músculo temporal de su fosa (Figs. 2 A y 2 B), e incluso desplazarlo lateralmente en su totalidad para poder tener buen acceso a la fosa temporal y pared lateral de la órbita. La osteotomía se realiza con material rotatorio preferentemente aunque también con sumo cuidado puede utilizarse el escoplo. El fragmento de hueso orbitario osteotomizado suele ser retirado, en cuyo caso se sumerge en suero fisiológico o solución antiséptica, o bien puede dejarse pediculado a través de las inserciones del músculo temporal. Una vez terminada la exéresis (Fig. 2 C) se recoloca el hueso osteotomizado y se fija mediante osteosíntesis reabsorbible o de titanio (Fig. 2 D). No se lleva a cabo ninguna osteosíntesis en caso 


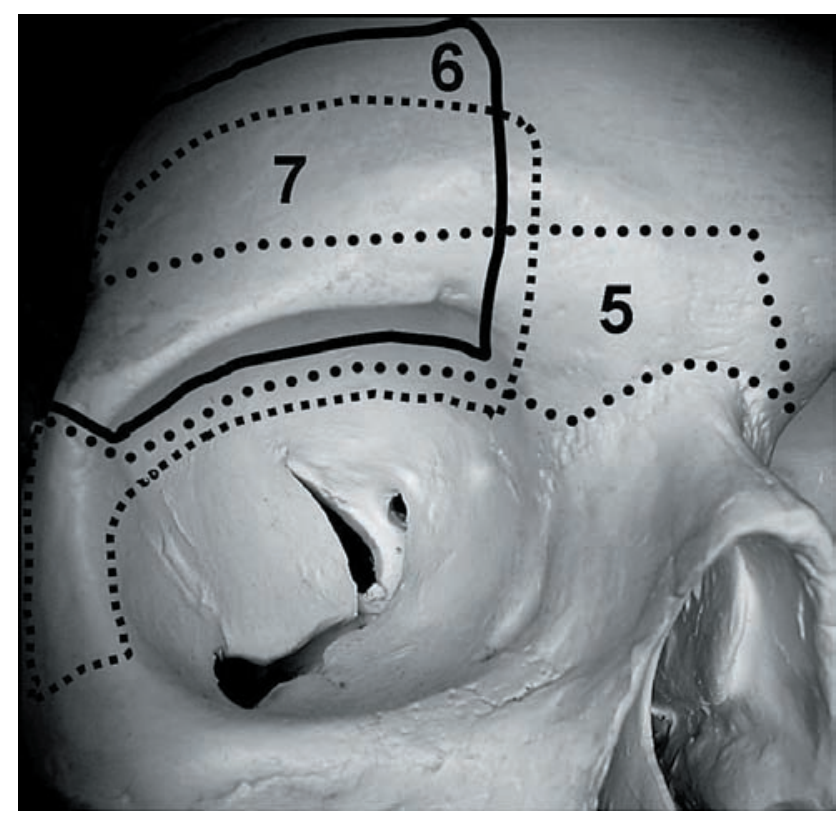

Figura 3. Esquema de orbitotomías mediante abordajes combinados o mixtos:(5) orbitotomía superior con extensión a la pared anterior del seno frontal, (6) orbitotomía superior con extensión intracraneal, y (7) orbitotomía superolateral con ostectomía del techo de la órbita.

de descompresión orbitaria, ostectomías o cuando por la localización tumoral la recolocación del fragmento óseo no tuviese trascendencia funcional o estética. Una vez comprobada la correcta adaptación de los tejidos blandos sobre el complejo reconstruido, se realiza comprobación de la hemostasia, colocación de dos drenajes aspirativos y cierre de la incisión de abordaje coronal por planos.

En la orbitotomía medial se realiza el abordaje coronal combinado con incisiones anteriores palpebrales. El ligamento cantal interno insertado en la cresta lacrimal anterior y posterior debe ir pegado a la pared de la osteotomía. Garantiza un buen acceso a la pared medial retroecuatorial, así como a las celdas etmoidales anteriores y posteriores. La debilidad y fragilidad de la pared medial de la órbita hace que en ocasiones se haga una ostectomía de esta pared, indicada en la cirugía descompresiva. La orbitotomía inferior para acceso al suelo de la órbita se puede realizar mediante incisiones en el párpado inferior, a veces acompañadas de un abordaje trans-antral. El límite posterior de la osteotomía del suelo de órbita es el límite inferior de la hendidura esfenoidal. El abordaje trans-antral permite acceder al suelo de la órbita y descender al globo ocular dentro del antro, aunque este abordaje es limitado.

Se utilizan además otras variantes técnicas de orbitotomías mediante abordajes combinados o mixtos (Fig. 3), como la orbitotomía superior con extensión a la pared anterior del seno frontal (con o sin osteoplasia frontal), la orbitotomía

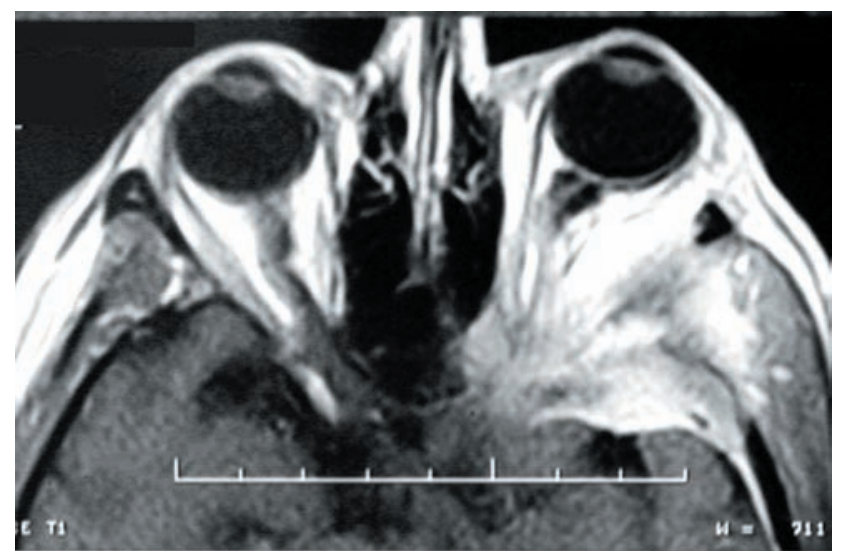

Figura 4 A. Caso 2. RNM de una lesión fronto-orbitaria que corresponde a un meningioma meningoendotelial.

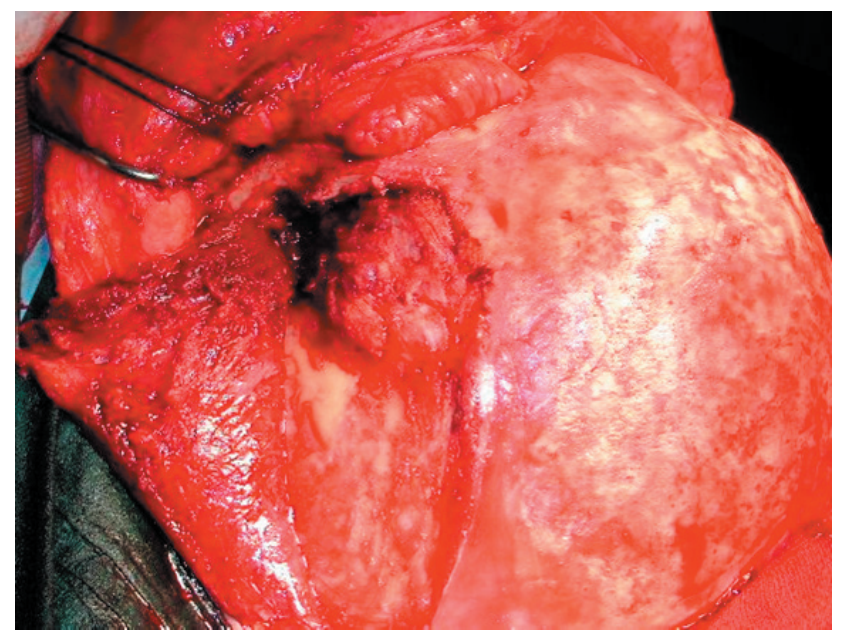

Figura 4 B. Caso 2. Detalle del abordaje coronal, acceso a la órbita y despegamiento del músculo temporal.

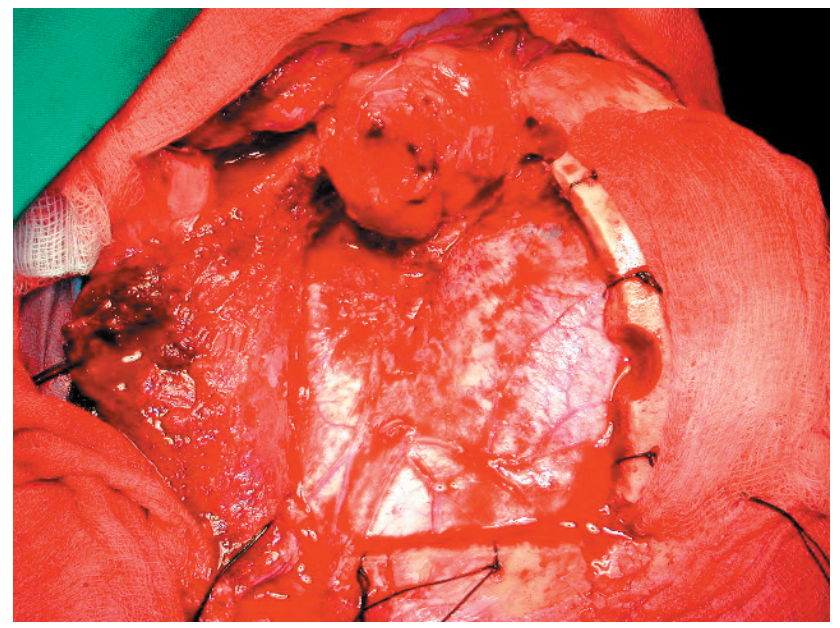

Figura 4 C. Caso 2. Exéresis del tumor mediante una orbitotomía supero-lateral acompañada de una vía neuroquirúrgica con craneotomía cuadrangular. 


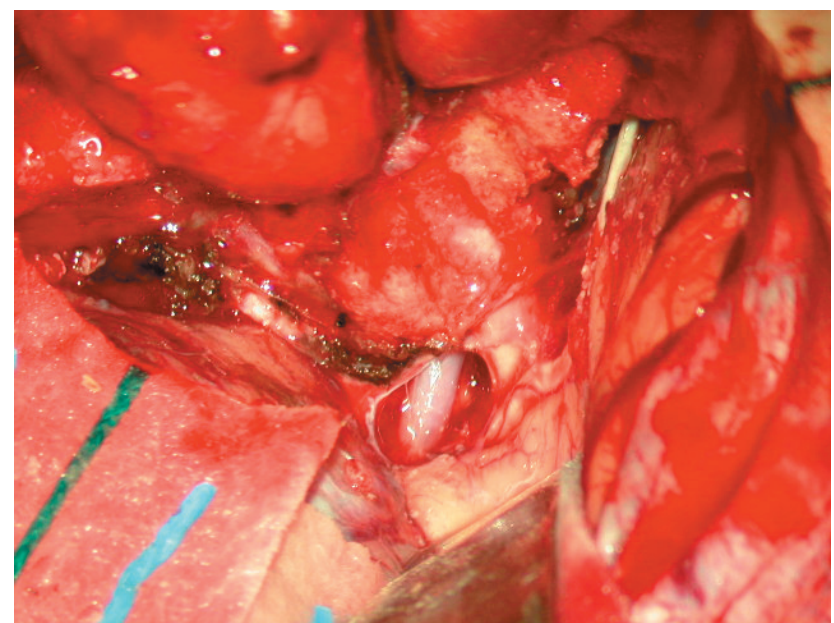

Figura 4 D. Caso 2. Control del tumor con disección extra e intradural exponiendo el nervio óptico en el canal orbitario.

superior con extensión intracraneal y la orbitotomía superolateral con ostectomía del techo de la órbita. Mediante los abordajes combinados intra/extracraneales se pretende acceder de forma combinada tanto a la órbita como a la cavidad intracraneal. La mayoría de los diseños de las osteotomías incluyen en una sola pieza los rebordes orbitarios superiores y la craneotomía fronto-temporal, obteniéndose una sola pieza en mono-bloque. Como alternativa puede extraerse también en dos piezas, la craneal y el reborde orbitario. El abordaje intracraneal puede plantearse para la remoción tumoral, para el control de la duramadre cuando se expone la masa encefálica por otro tipo de abordaje o para el control del nervio óptico a nivel más posterior (Fig. 4 A). Para la resección de la lesión, el despegamiento se hará intraduralmente si la duramadre está afectada, o extraduralmente si no lo está (Figs. $4 \mathrm{~B}$ y 4 C). La osteotomía del techo orbitario anterior al canal óptico se realiza extraduralmente, y permite el acceso a los compartimentos superiores más posteriores de la órbita (Fig. 4 D).

\section{Resultados}

Las indicaciones de las orbitotomías fueron: exéresis tumoral en 75 pacientes $(86,20 \%)$, biopsia diagnóstica en 2 $(2,30 \%)$ y descompresión orbitaria en $10(11,50 \%)$. En todos los casos se empleó un abordaje coronal, que se acompañó en 6 casos de incisiones anteriores (4 subciliares inferiores y 2 transconjuntivales superiores con cantotomía lateral). El despegamiento del músculo temporal se llevó a cabo en 81 pacientes $(93,10 \%)$. La orbitotomía más utilizada fue la lateral o superolateral en 76 casos $(87,35 \%)$, seguida de la orbitotomía medial (4 casos), combinada/mixta (4 casos)
Tabla 4

Complicaciones postoperatorias

\begin{tabular}{lcc}
\hline & $\mathrm{n}$ & $\%$ \\
\hline Diplopia temporal & 8 & 24,24 \\
Hematoma & 8 & 24,24 \\
Ptosis palpebral temporal & 7 & 21,21 \\
Ptosis palpebral permanente & 6 & 18,18 \\
Oftalmoparesia pupilar & 6 & 18,18 \\
Oftalmoparálisis pupilar & 5 & 15,15 \\
Cefalalgia / Algia facial atípica & 5 & 15,15 \\
Infección / Celulitis periorbitaria & 4 & 7,27 \\
Fístula de LCR & 2 & 3,64 \\
Paresia frontal & 2 & 3,64 \\
Retirada material osteosíntesis & 2 & 3,64 \\
Hidrocefalia & 1 & 1,82 \\
Carcinomatosis meníngea & 1 & 1,82 \\
Ictus masivo & 1 & 1,82 \\
Queratitis & 1 & 1,82 \\
Shock séptico & 1 & 1,82 \\
Neuropatía óptica & 1 & 1,82 \\
Atrofia músculo temporal & 1 & 1,82 \\
Fístula palatina & 1 & 1,82 \\
Parálisis frontal & 1 & 1,82 \\
Diplopia definitiva & 1 & 1,82 \\
Reabsorción parcial orbitotomía & 1 & 1,82 \\
\hline
\end{tabular}

y superior ( 3 casos). Se llevó a cabo ostectomía en mayor o menor medida en 10 casos $(11,50 \%)$, en 5 orbitotomías mediales para descompresión orbitaria, y en 3 mediales, 1 inferior y 1 superior por exéresis tumoral. En 79 orbitotomías $(90,80 \%)$ se realizó algún tipo de osteosíntesis (45 casos con material bio-reabsorbible, 31 casos con mini/ microplacas de titanio y 3 casos con miniplacas y alambre). En 5 casos $(5,75 \%)$ se colocaron injertos óseos tomados de la calota craneal.

La duración media de las intervenciones fue de 3 horas y 27 minutos. No se presentó ninguna complicación peroperatoria de gravedad que impidiera realizar la intervención planificada. La más frecuente fue la exposición o rotura de la duramadre en 10 pacientes $(11,50 \%)$. Otras incidencias peropertorias destacables fueron: bradicardia o asistolia en 6 pacientes $(6,90 \%)$, transfusión de hemoderivados en 4 pacientes $(4,60 \%)$ y sondaje de la vía lacrimal en 2 pacientes $(2,30 \%)$. Se recogieron 66 complicaciones postoperatorias en 22 pacientes (Tabla 4). 45 complicaciones fueron transitorias y 21 permanentes, teniendo mal pronóstico un ictus masivo, un shock séptico y una diplopia definitiva. El tiempo medio de estancia postoperatoria fue 
de 7,4 días (3-30 días), que se prolongaron en un paciente por una infección por Acinetobacter multirresistente a antibióticos y en 3 pacientes por fístulas de LCR. Hubo que reintervenir en 4 casos por complicación ( 2 fístulas de LCR y 2 retiradas de material de osteosíntesis intolerado).

En 15 pacientes $(17,25 \%)$ se requirió colaboración multidisciplinar con neurocirugía. 1 paciente precisó una indicación urgente por compromiso de la agudeza visual por un hemolinfangioma infantil. El seguimiento mínimo de los tumores ha sido de 36 meses. Se ha detectado enfermedad residual o metastásica en 10 casos $(11,50 \%)$. La mortalidad en nuestra serie ha sido de 4 pacientes (2 rabdomiosarcomas, 1 meningioma endotelial y 1 linfoma no Hodgkin).

\section{Discusión}

A pesar de que se han introducido nuevas técnicas y procedimientos para acceder a la órbita (como la endoscopia $^{4,6}$, cada vez más ampliamente utilizada para lesiones y tumores adyacentes a los senos etmoidal y maxilar), el abordaje coronal acompañado de una orbitotomía supero/ lateral sigue siendo la técnica más utilizada por la mayoría de los autores $3,4,8,9,17,19,21$, especialmente para la extirpación (o biopsia) de lesiones extradurales laterales al nervio óptico (tanto intra como extraconales) y de la glándula lacrimal. La indicación de cada abordaje variará según la localización, tamaño de la lesión, intención de la cirugía (extirpación, biopsia o descompresión) y las características histológicas. En la patología tumoral orbitaria hay que considerar los procesos neoplásicos de naturaleza benigna o maligna y los que sin ser tumores tienen un comportamiento expansivo similar ${ }^{22,27}$. En nuestra serie, se recogieron 14 tumores de naturaleza benigna $(16,10 \%)$ con comportamiento localmente agresivo y otros 10 procesos expansivos no tumorales $(11,50 \%)$.

Para llevar a cabo una orbitotomía ósea, el abordaje coronal se caracteriza por ofrecer un buen campo quirúrgico, con un óptimo resultado estético. Es fácil y seguro de realizar, y permite realizar cómodamente las osteosíntesis y ofrece la posibilidad de tomar injertos óseos. Según nuestros resultados y siguiendo a otros autores ${ }^{17,23}$, la incisión coronal, acompañada en su caso de incisiones anteriores, es la idónea para la realización de las osteotomías orbitarias salvo en aquellos casos que sólo se deban abordar por vía anterior. Los cuadrantes mediales pueden abordarse tanto por vía coronal como por incisiones paralateronasales o mediocantales.

En nuestra serie, la técnica quirúrgica empleada para el abordaje coronal no difiere de la descrita por Abubaker y cols. ${ }^{1}$ salvo que a nivel de la región temporal preferimos incidir las fascias temporales siguiendo la línea temporal, lo cual permite poder despegar el músculo temporal de forma completa y poder acceder con mayor garantía a la región temporal y lateral de la órbita. La movilización completa del músculo temporal de su fosa puede provocar algún grado de atrofia postoperatoria ${ }^{17}$, que puede minimizarse si el músculo sólo se despega parcialmente y si posteriormente se sutura la fascia en su línea de inserción. Como complicación a esta técnica se ha descrito el trismus postoperatorio ${ }^{2,23}$, que en nuestra casuística no se ha presentado en ningún caso. Ante una paresia y/o parálisis frontal hay que tomar una actitud expectante ya que en la mayoría de las ocasiones se resuelve de forma espontánea en el plazo de unas semanas. En 2 ocasiones apareció una paresia frontal transitoria que recuperaron antes de las 9 semanas. En 1 paciente intervenido de un glioma óptico ha persistido una parálisis frontal permanente, a pesar de haber realizado la técnica correctamente.

Las orbitotomías se planificaron de tal forma que pudieran garantizar un acceso amplio y seguro, y el tratamiento correcto según el caso. El diseño de la osteotomía utilizada dependió de la localización, extensión y naturaleza de la lesión, y requirió la colaboración habitual con neurocirugía. Las orbitotomías óseas lateral y supero-lateral fueron empleadas en 76 casos (87,35\%), especialmente en casos de localización retrobulbar extraconal, aunque también para los intraconales, abordados mediante incisión radial de la periórbita y cápsula de Tenon a nivel lateral. Consideramos que la vía intracraneal extradural debe reservarse para lesiones cerca del ápex ${ }^{26}$, el cual es difícil de abordar mediante otra vía, puesto que para el resto de localizaciones es preferible el uso de los diseños de orbitotomías óseas dado que el campo quirúrgico es mayor. La realización de una orbitotomía ósea junto con una craneotomía frontotemporal no solo mejora el acceso quirúrgico sino que también favorece que la retracción del cerebro sea menor ${ }^{4}$. Cuando la afectación del techo de órbita se sitúa a nivel anterior puede igualmente abordarse por vía extracraneal.

El grado de reabsorción ósea, tanto de las osteotomías orbitarias practicadas como del material óseo injertado, suele ser de mínima cuantía y escasa consideración. Algunos autores suelen utilizar técnicas de marginotomías superolaterales pediculadas al músculo tempora ${ }^{14}$. Según nuestra opinión no es del todo necesario dicha modalidad al tratarse de un hueso fácilmente adaptable al defecto, poseer idénticas características que el hueso circunscrito y poderse someter a una óptima fijación con los métodos de osteosíntesis. En nuestra serie, la reabsorción no fue relevante, y sólo en 5 casos $(5,75 \%)$ obtuvimos reabsorciones parciales de los fragmentos osteotomizados reposicionados.

En 79 casos $(90,80 \%)$ se realizó algún tipo de osteosíntesis, siguiendo los principios básicos de la traumatología y cirugía reconstructiva craneofacial. Los resultados han sido plenamente satisfactorios con todos los sis- 
temas de osteosíntesis, aunque se aprecia una preferencia actual hacia los sistemas bio-reabsorbibles. Los objetivos principales que persiguen los sistemas de osteosíntesis en la cirugía de la órbita son: precisión para conseguir la reposición del fragmento óseo, semi-rigidez al tratarse de huesos no sometidos a fuerzas musculares de consideración, y reducción del tamaño y grosor para evitar intolerancias y defectos estéticos o que sean biodegradables. No se empleó ninguna modalidad de osteosíntesis en 8 casos del total de la serie $(9,20 \%)$, cuando la posible reabsorción o no recolocación del fragmento osteotomizado no tenía trascendencia funcional y/o estética, en casos de descompresión orbitaria o cuando se realizó ostectomía ósea.

Cuando se necesitó una fijación semirrígida o un reposicionamiento del fragmento osteotomizado se optó por una osteosíntesis con miniplacas y microplacas de titanio. Sin embargo, la aparición en el mercado del material bioreabsorbible como sistema de osteosíntesis ha supuesto una excelente alternativa al titanio para la fijación ósea semirrígida craneomaxilofacial ${ }^{5}$, sobre todo en pacientes pediátricos porque no es necesario retirarlos ni interfieren con el crecimiento craneofacial ${ }^{24}$. Así, en nuestra serie es el método de osteosíntesis más utilizado desde el comienzo de su uso a partir de 1998, en un total de 45 casos (51,72\%). El material utilizado ha sido un compuesto de dos copolímeros de ácido L-láctico y ácido glicólico en proporción 82:18 (LactoSorb ${ }^{\circledR}$ ) y de $1,5 \mathrm{~mm}$ de grosor que conserva su resistencia al menos el $70 \%$ del total durante al menos 6-8 semanas y un tiempo de degradación variable entre los 9-15 meses. Como desventajas se han descrito ${ }^{18}$ la intolerancia, biodegradación prolongada en algunos compuestos, migración del material, grosor de las placas, falta de control radiológico, necesidad de una óptima cobertura tisular y elevado coste de estos biomateriales, si bien se obvian segundas intervenciones para su retirada. Se retiró el material de osteosíntesis en 2 pacientes. Una miniplaca de titanio en un paciente con antecedentes previos de cirugía y radioterapia, y una placa bio-reabsorbible en una paciente de edad media intervenida de un mucocele frontoorbitario, quien debutó con una celulitis orbitaria a los 3 meses de la intervención.

Se han descrito numerosas complicaciones peroperatorias en la cirugía de la órbita ${ }^{8}$ : hemorragias de difícil control, sección de pares craneales, bradicardia y parada cardíaca, exposición y rotura de la duramadre, lesión de las vías lacrimales, lesión de los ligamentos cantales, lesión de la musculatura extraocular, lesión de estructuras palpebrales, fractura de alguna pared orbitaria y comunicaciones sino-orbitarias, entre otras. Algunas de estas complicaciones o incidencias son previsibles, otras aparecen accidentalmente pudiendo llegar a variar la planificación prevista. En la serie aportada se han registrado 22 complicaciones o incidencias peroperatorias, aunque ninguna de ellas impi- dió realizar la intervención programada y planificada.

En todos los pacientes se les hizo una profilaxis antibiótica. En 81 de los 87 casos se utilizó la pauta consistente en 1 gramo de amoxicilina-ácido clavulánico administrada vía parenteral intravenosa en la inducción anestésica, repitiendo las dosis cada 3 horas según la duración de la intervención. En 3 pacientes se optó por clindamicina a dosis de $600 \mathrm{mg}$ por vía intravenosa y en los 3 casos restantes, cefazolina a dosis de 1 gramo por vía intravenosa. Este tipo de cirugía suele considerarse como una cirugía limpia con escasa probabilidad de infección ${ }^{13}$. Pese a ello, aparecieron infecciones en el $7.27 \%$ de los casos (4 pacientes). El mayor riesgo radicó en aquellos casos donde se comunicó la zona quirúrgica con el exterior (exenteración) y a nivel de la incisión bicoronal.

Las complicaciones postoperatorias representan un indicador fundamental para valorar el resultado e idoneidad de la cirugía de la órbita. Dejando a un lado las complicaciones comunes a cualquier cirugía (infección, mutilación, cicatrices ... ), hay que considerar una serie de complicaciones «específicas» que hacen referencia a diversos aspectos ${ }^{8}$ : hematoma retrobulbar, síndrome de ápex orbitario y hendidura esfenoidal, posibles secuelas sobre la función de la visión (cataratas, queratoconjuntivitis, pérdida de agudeza visual ... ), lesión de la musculatura extraocular (diplopia) y palpebral (ptosis, entropión, ectropión), alteración de la estética facial por falta de crecimiento orbitario, etc. El análisis de nuestra serie puso de manifiesto un total de 66 complicaciones postoperatorias en las 87 orbitotomías realizadas. Consideramos 3 complicaciones de mal pronóstico (ictus masivo, shock séptico y afectación del nervio óptico con ptosis definitiva).

Se presentaron dos fístulas de LCR tras la extirpación de un ganglioneuroblastoma y de un rabdomiosarcoma que precisaron tratamiento quirúrgico dado que no cerraron de forma espontánea y existían signos de infección a nivel locorregional. El primer caso se trató mediante la utilización de injerto de fascia lata autóloga, junto con adhesivos biológicos y drenaje lumbar, y el segundo mediante limpieza de la cavidad orbitaria, sutura y adhesivos biológicos. El resultado en ambos casos fue excelente y sin secuela alguna. La ptosis palpebral transitoria o de carácter temporal cursó en 7 casos, desapareciendo en un plazo que varió desde 10 días hasta 13 semanas. Su persistencia representa una complicación importante dada las importantes secuelas funcionales y estéticas. En 6 casos que se precisó una extirpación de estructuras musculares y nerviosas del párpado superior, se detectó una ptosis permanente. La diplopia es una complicación que puede presentar importantes secuelas funcionales y estéticas y cuyo tratamiento tiene unos pobres resultado ${ }^{11}$. En nuestro estudio, 8 casos presentaron una diplopia postquirúrgica de forma temporal, quedando resuelta en un intervalo entre 1-7 semanas. En 1 caso ha 
quedado una diplopia permanente tras la extirpación de una lesión intraconal. El edema importante y la contusión muscular y nerviosa, pueden explicar la aparición de la mayoría de las diplopias postquirúrgicas, pero ante la sospecha o certeza de incarceración muscular postquirúrgica, hace necesaria la cirugía exploradora.

En 15 enfermos (17,25\%) se planteó un enfoque interdisciplinario para conseguir óptimos resultados, en 4 pacientes con afectación del SNC y 5 pacientes con extensión a seno y hueso frontal, 3 seno esfenoidal y 3 celdillas etmoidales. El objetivo principal es la labor coordinada entre las distintas especialidades que garanticen la calidad asistencial en el diagnóstico, abordaje quirúrgico y la exéresis, así como en el manejo del pre, intra y postoperatorio $^{4,7,20}$.

\section{Conclusiones}

La mayor parte de los tumores y lesiones intraorbitarias extradurales se pueden abordar mediante una incisión coronal que permite realizar con seguridad y garantía cualquier tipo de orbitotomía ósea, combinando un óptimo resultado estético y funcional, excepto para el abordaje del suelo de la órbita que se debe llevar a cabo por vía anterior. Las orbitotomías lateral y superolateral proporcionan un buen abordaje quirúrgico al espacio retrobulbar. El acceso al ápex, canal orbitario y zona medial al nervio óptico requiere comúnmente un abordaje combinado neuroquirúrgico a través de fosa anterior. La región orbitaria de más difícil acceso es la región retrobulbar intraconal inferior. La osteosíntesis bio-reabsorbible como método de fijación semirrígida de las orbitotomías ha supuesto una alternativa al material de titanio, incluso de elección en la edad pediátrica, aunque existen discrepancias aún no resueltas en cuanto a las ventajas e inconvenientes que presenta con respecto a la osteosíntesis clásica. La morbilidad de esta cirugía es baja, siendo lo más frecuente la aparición de complicaciones nerviosas (diplopia, ptosis, etc.) que se resuelven de forma espontánea en la mayoría de las ocasiones en un corto intervalo de tiempo.

\section{Bibliografía}

1. Abubaker, A.O., Soteranos, G., Patterson, G.T.: Use of the coronal surgical incision for reconstruction of severe craniomaxillofacial injuries. J Oral Maxillofac Surg 1990; 48: $579-586$.

2. Al-Mefty, O., Anand, V.K.: Zygomatic approach to the skull base lesions. J Neurosurgery 1990; 73: 668-731.

3. Arai, H., Sato, K., Katsuta, T., Rhoton, A.L.: Lateral approach to intraorbital lesions: Anatomic and surgical considerations. Neurosurgery 1996; 39: 1157-1163.

4. Bejjani, G.K., Cockerham, K.P., Kennerdel, J.S.,
Maroon, J.C.: A reappraisal of surgery for orbital tumors. Part I: extraorbital approaches. Neurosurg Focus 2001; 15; 10: E2.

5. Bell, R.B., Kindsfater, C.S.: The use of biodegradable plates and screws to stabilize facial fractures. J Oral Maxillofac Surg 2006; 64: 31-39.

6. Burson, J.G., Gussack, G.S., Hudgins, P.S.: Endoscopic approach to the pediatric orbit. Laryngoscope 1995; 105: 771773.

7. Cockerham, K.P., Bejjani, G.K., Kennerdell, J.S., Maroon, J.C.: Surgery for orbital tumors. Part II: transorbital approaches. Neurosurg Focus 2001; 15; 10: E3.

8. Espín, F., Gutiérrez, J.L., Infante, P., Marín, R., Mayorga, F., García Perla, A. Protocolo y planificación de las orbitotomías óseas controladas en los tumores y tumoraciones orbitarias. Rev Esp Cir Oral y Maxilofac 1996; 18: 26-39.

9. Herencia Nieto, H., Verdaguer Martín, J.J., Riba García, F., Calvo de Mora Álvarez, F., del Amo Fernández de Velasco, A., Pujol Romanya, R., Navarro Cuellar, C.: Orbitotomía lateral mediante abordaje temporal. Rev Esp Cir Oral y Maxilofac 2005; 27: 335-343.

10. Kalmann, R., Mourits, M., van der Pol, J.P., Koornneef, L.: Coronal approach for rehabilitative orbital decompression in Graves' ophthalmopathy. Br J Ophthalmol 1997; 81: 41-45.

11. Kennerdell, J.S., Maroon, J.C., Malton, M.L.: Surgical approaches to the orbital tumors. Clin Plast Surg 1988; 15: 273-277.

12. Leone, C.R., Wissinger, J.P.: Surgical approaches to the diseases of the orbital apex. Opbthalmology 1988; 95: 391-397.

13. Mangram, A.J., Horan, T.C., Pearson, M.L., Silver, L.C., Jarvis, W.R.: The Hospital Infection Control Practices Advisory Committee.Guideline for the prevention of surgical site infecction,1999. Infect Control Hosp Epidemiol 1999; 20: 250-278.

14. Maroon, J.C., Kennerdell, J.S.: Surgical approaches to the orbit. Indications and techniques. J Neurosurg 1984; 60: 1226-1231.

15. Mohr, C., Schettler, D., Heesen, J.: The surgical approach to orbital space -occupying lesions. J Craniomaxillofac Surg 1989; 17: 149-154.

16. Natori, Y., Rhoton, A.L.Jr.: Transcranial approach to the orbit: microsurgical anatomy. J Neurosurg 1994: 81: 7886.

17. Paolini, S., Santoro, A., Missori, P., Pichierri, A., Esposito, V., Ciappetta, P.: Surgical exposure of lateral orbital lesions using a coronal scalp flap and lateral orbitozygomatic approach: clinical experience. Acta Neurochir (Wien) 2006; 148: 959-963.

18. Pensler, J.M.: Role of resorbable plates and screws in craniofacial surgery. J Craniofac Surg 1997; 8:129-134.

19. Rothon A.L. Jr.: The orbit. Neurosurgery 2002; 51: 
303-34.

20. Scheuerle, A.F., Steiner, H.H., Kolling, G., Kunze, S., Aschoff, A.: Treatment and long-term outcome of patients with orbital cavernomas. Am J Ophthalmol 2004; 138: 23744.

21. Schick, U., Dott, U., Hassler, W.: Surgical treatment of orbital cavernomas. Surg Neurol 2003; 60: 234-244.

22. Shields, J.A., Shields, C.L., Scartozzi, R.: Survey of 1264 patients with orbital tumors and simulating lesions: The 2002 Montgomery Lecture, part 1. Ophthalmology 2004;111: 997-1008.

23. Stewart, W.B., Levin, P.S., Toth, B.A.: Orbital surgery. The technique of coronal scalp flap approach to the lateral orbitotomy. Arch Ophthalmology 1988; 106: 17241726 .

24. Tharanon, W., Sinn, D.P., Hobar, P.C., Sklar, F.H., Salomon, J.: Surgical outcomes using bioabsorbable plating systems in paediatric craniofacial surgery. J Craniofac Surg 1998; 9: 441-444.
25. Tsirbas, A., Kazim, M., Close, L.: Endoscopic approach to orbital apex lesions. Ophthal Plast Reconstr Surg 2005; 21: 271-275.

26. Viale, G.L., Pau, A.: A plea for postero-lateral orbitotomy for microsurgical removal of tumours of the orbital apex. Acta Neurochir 1998; 90: 124-126.

27. Weisman, R.A., Kikkawa, D., Moe, K.S., Osguthorpe, J.D.: Orbital tumors. Otolaryngol Clin North Am 2001; 34: 1157-1174.

Infante-Cossío, P; Molina-Martínez, M.; García-Perla, A.; Espín-Gálvez, F.; Romero Arce, J.; Gutiérrez-Pérez, J.L.: Orbitotomías óseas mediante abordaje coronal: estudio retrospectivo de 87 lesiones intraorbitarias. Neurocirugía 2008; 19: 322-331.

Correspondencia postal: Dr. Pedro Infante Cossío. Servicio de Cirugía Maxilofacial. Hospital Universitario Virgen del Rocío. Av. Manuel Siurot s/n. 41013-Sevilla (Spain). 